\title{
Cell Concentration and Separation in the Field of a Standing Ultrasonic Wave for Medicine and Biotechnology
}

\author{
Diana G. Sadikova, Timofey N. Pashovkin \\ Institute of Cell Biophysics, Russian Academy of Sciences, Pushchino, Russia \\ Email: sdg7@list.ru
}

Received December 28, 2012; revised January 30, 2013; accepted February 11, 2013

\begin{abstract}
This work is carried out to determine the empirical boundary conditions of concentration/separation of yeast cells, red blood cells of rats and guinea pigs in standing ultrasonic wave, based on the performed theoretical calculations. The results indicated that increase flow rate of the medium above the theoretically calculated values of cell retention in a standing ultrasonic wave causes them to wash away from the camera separation ultrasound.
\end{abstract}

Keywords: Ultrasound; Separation; Concentration; Acoustic Filter

\section{Introduction}

In the past few years, more and more ultrasonic methods are employed as filters in medicine and biotechnology. Filters of this type are able to effectively delay the different particles (for example, cells) in variable-pressure nodes. The basic mechanisms of this acoustic filter are associated with various forces in a standing ultrasonic wave on the cells in suspensions. In the last few years, the possibility of cell redistribution in a volume under these forces led to the development of several new methods of cells concentration and separation in the field of a standing ultrasonic wave both for medicine, biotechnology, and cytobiology.

\section{Basic Concepts of Methods of Ultrasonic Concentration and Separation of Suspension Particles}

Currently, there are many concepts on the separation and concentration of suspended particles of different origin under the forces acting on these particles in ultrasonic fields. They can be classified into categories based on the use of 1) standing or running acoustic waves; 2) modes with continuous and periodically stopped medium flows; and 3) resonant or non-resonant conditions. A specific system can include any of the above combinations; however, all practical methods are based on the action of the radiation pressure force (radiation force) on cells. Moreover, other forces can also be used, depending on the design of the device intended for ultrasonic selection.

The effect of particle coagulation in the standing ul- trasonic wave has long been known and studied by many authors [1-3]. Under the action of the radiation force, particles are collected in planes perpendicular to the direction of ultrasound propagation [3]. Then, the collected particles are concentrated by acoustic forces. As the ultrasonic field is turned off, large agglomerates emerge or precipitate by gravity. Such a coagulation method can be applicable only to particles forming stable agglomerates. Otherwise, agglomerates are decomposed after ultrasound turning off.

Another method using the standing ultrasonic wave consists in that the radiation force displaces particles in the plane of nodes of a standing ultrasonic wave between the transducer and reflector. The reflector wall is equipped with many small outlets arranged in the regions of distributions of the particle layer. The laminar flow parallel to the planes of particle collection moves particles to their outlet. This method does not require particle agglomeration and allows continuous operation. However, such a method works if planes of particle collection are steadily retained in space. Since the ultrasound velocity in liquid depends on temperature, the ultrasonic wavelength, hence, the coordinate of planes of particle collection change with temperature if the operating frequency is constant. Therefore, stable operation of devices based on the above method requires precise temperature stabilization or frequency tuning to compensate for variations of ultrasound velocity during long-term operation. Basic techniques that use the same principles have been described by the authors [4-8].

Peterson et al. [9] described a method of particle se- 
paration based on drifting acoustic fields, using which, a certain displacement of particles to certain regions within the separation chamber is achieved. In this case, the term "drifting field" refers to pseudo-standing acoustic waves with node planes slowly moving in space. Such a drifting field can be produced by superposition of two counter running waves slightly differing in frequency. The method uses 1) a waveguide with emitters at both ends, excited at different frequencies; 2) one active emitter excited with a continuously varying frequency and a fixed reflector; 3) an emitter operating at a fixed frequency and a moving reflector; and 4) frequency deviation and a synchronized moving reflector.

Despite the significant number of papers devoted to particle separation using ultrasound, most studies were restricted to the confirmation of operability of the main methods or the development of laboratory-scale devices. Nevertheless, it was shown that the especially high potential for successful application of acoustic separation methods exists in the field of biotechnology [10-12].

In this study, we used a laboratory prototype of the longitudinal ultrasonic selection system for separating and concentrating cells and particles in the flow passing through the cell, in which the standing wave is generated. It includes a thermostating system for maintaining a constant temperature. Simultaneously, the thermostating liquid is a contact medium between the emitter and cell. The main difference of this system from the considered above is that the field of the standing ultrasonic wave in the chamber is formed due to interference of wave's incident at different angles. Currently, a device with improved, in comparison with our system, characteristics was developed at the Institute for Analytical Instrumentation, Russian Academy of Sciences, St. Petersburg.

\section{Aim of This Study}

The above ultrasonic separation systems can operate without suspension flows for particle coagulation or in the mode of continuous suspension flows for particle separation and concentration. Flow-through systems are more advanced, since it is easier to maintain a stable temperature in them, and they provide a higher separation percentage due to conditions of operation.

Cell concentration in the flow-through system depends on the combined effect of forces induced in the ultrasonic field and due to the liquid flow around particles confined by the field. To make it possible to predict the cell behavior in the field of the standing ultrasonic wave using such systems, a number of physical parameters of cells and cultivation media, which enter the formulas for calculating the radiation, Stokes, Bjerknes, Bernoulli, and gravitational forces acting on cells, should be comprehensively studied. Previously, we calculated the forces acting on cells in suspensions in the field of the standing ultrasonic wave and determined about fifteen parameters of the field, cells, and suspending media, which can affect cell exfoliation, separation, and concentration in such fields [13,14-16]. Also, we determined of the concentration, separation, and washout regions for three cell types in the coordinate system of the average energy density of the standing ultrasonic wave and the linear velocity of the cell suspension flow. Been shown theoretically calculated region separating lines of concentration/separation for: yeast cells, rat erythrocytes, and guinea-pig erythrocytes [13]. This work is performed to confirm in experiments the theoretical calculations of the boundary conditions of concentration/separation to study cells in a standing ultrasonic wave. This is necessary to obtain a more complete picture of the process of concentration/separation cells depending on the different rates of flow of the medium.

\section{Materials and Methods}

\subsection{Objects of Study}

As objects of study, we used rat erythrocytes, guinea-pig and yeast (Saharomices cerevisiae) cells. Choice of objects of study are explained the difference their size and shape. This allowed us, in a previous study, to consider the forces acting on the cells of simple shape (sphere and disk) [13].

Wistar rat erythrocytes were obtained from a caudal vein. Blood was placed into a $0.9 \% \mathrm{NaCl}$ physiological solution with heparin addition. Then, cells were triply washed and diluted in $0.9 \% \mathrm{NaCl}$ to a required concentration. The contents of the dry weight of the cell and water in it were $9 \%$ and $94 \%$, respectively.

Guinea-pig erythrocytes were obtained from the blood of an ear vein, which was placed into a $0.9 \% \mathrm{NaCl}$ physiological solution with heparin addition. Then, cells were triply washed and diluted in $0.9 \% \mathrm{NaCl}$ to a required concentration. The contents of the dry weight of the cell and water in it were $14 \%$ and $86 \%$, respectively.

Yeast cells were obtained from a dry Saharomices cerevisiae yeast culture. It was diluted in distilled water to prevent vigorous cell reproduction and to obtain a stable cell concentration in suspension. At such a dilution, the cell concentration did not exceed $1-2 * 10^{9}$ cells $/ \mathrm{ml}$.

The contents of the dry weight of the cell and water in it were $25 \%$ and $75 \%$, respectively [17]. The cell size was determined by light microscopy. To this end, the cells in the Goryaev chamber were photographed using a video ocular. Then, the cell diameter was measured. In the case of erythrocytes, not only the outer diameter, but also the diameter of the biconcave membrane was measured. 


\subsection{Longitudinal Ultrasonic Selection Method}

The separation and concentration method is based on the principle called by its author N. N. Knyaz'kov [18,19] as longitudinal ultrasonic selection (LUS) of suspensions. It consists in that cells are confined by the field of the standing ultrasonic wave during suspension flow along the direction of ultrasound propagation. In this case, particles in the standing wave's field are affected by various forces, i.e., the radiation and Stokes forces, the force associated with the liquid flow, the gravitational force, and the forces of the interaction between particles, i.e., the Bjerknes and Bernoulli ones.

To provide an efficient implementation of the method, the ultrasonic wavelength is chosen from the relation $\lambda \leq$ $2 \pi a$, where $\lambda$ is the ultrasonic wavelength and is the characteristic size of particles displaced by the field. If this condition is violated, the use of the radiation force becomes inefficient.

Due to the complex dependence of the radiation force on the LUS system characteristics, the operation method cannot be described by a simple mathematical expression. Therefore, the liquid velocity and the intensity of the ultrasonic field are experimentally determined for each particular case.

The device for separating and concentrating a liquid dispersion system (Figure 1) contains chamber 1 with inlet and outlet tubes for pumping cell suspensions, which is placed into glass thermostating chamber 2 . The emitter with an operating frequency of $2.64 \mathrm{MHz}$ is installed in the end of chamber 2. Glass surface 3 at the other end of chamber 2 serves as a reflector; thus, the standing ultrasonic wave is generated. Two LUS systems were used a focusing transducer $4 \mathrm{~cm}^{2}$ in area, its radius of the focal spot and length of the focal region were $1.2 \mathrm{~mm}$ and 7 $\mathrm{mm}$, respectively.

Experimental determination of concentration/separation boundary conditions for tested cells in a standing ultrasonic wave. Determination of boundary conditions for

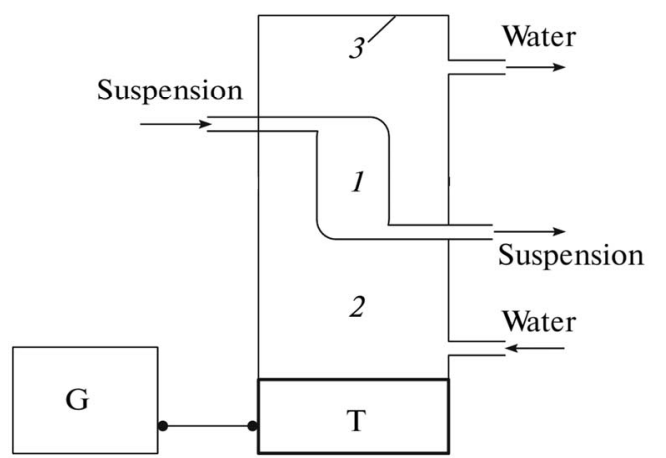

Figure 1. Device for longitudinal ultrasonic selection: (PS) power supply, (T) piezoceramic transducer, (G) ultrasonic generator, (1) thermostated chamber, (2) thermostating chamber, and (3) reflector. concentration/separation cells in the ultrasound field was performed using longitudinal ultrasonic device separation cells (Figure 1).

Concentration of cells was measured the in the chamber number 1 , with different rates of pumping cell suspension through the ultrasonic field at the average energy density of $1.6 \times 10^{-5} \mathrm{~J} / \mathrm{cm}^{3}$ and $2.5 \times 10^{-5} \mathrm{~J} / \mathrm{cm}^{3}$.

A peristaltic pump calibrated before each experiment was used to change the flow rate of cell suspension. Tubing of different diameter $(1-5 \mathrm{~mm})$ used to range a speed of the peristaltic pump. Cell suspension filled the chamber No. 1 (Figure 1). Subsequently ultrasonic field was superposed with specified pumping speed. Through the chamber passed a constant volume of the cell suspension $(1 \mathrm{ml}$ or $10 \mathrm{ml})$. Then, the suspension of the cells was decanted from of the chamber No. 1. The number of cells was calculated in Goryaev chamber. In this case, the number of cells was calculated in 5 or more large squares. For each type of cells was made at least five experiments. This resulted in a mean concentration of cells which are held in a standing ultrasonic wave, and their error of the mean.

\section{Results and Discussion}

In our system for the ultrasound concentration of cells in suspension the radiation pressure and the Stokes force are fundamental (under flow of the medium), so the gravitational force and the Bjerknes and Bernoulli forces was not considered, because these forces are very small or are responsible for the interaction of cells each other (for example, the force of the Bjerknes).

In the presence of flow of suspension is necessary to consider the movement of cells under the influence of radiation pressure and influence of the Stokes force on cells.

We investigated these two forces, and picked up the boundary conditions for the concentration and separation of cells in suspension in a standing ultrasonic wave, which allowed us to theoretically find the flow rate environment and the average energy density.

To obtain the boundary values of pumping rate in the chamber for ultrasonic concentration, it was necessary to compare the radiation force at different average densities of ultrasonic energy (for the cells) with the Stokes force. [13].

Subject to the condition that $F_{r} \geq F_{S 1}$ will be holding cells in an ultrasonic field $\left(\mathrm{F}_{\mathrm{r}}\right.$-radiation force, $\mathrm{F}_{\mathrm{S} 1}$-Stokes force).

To obtain the boundary values of the medium's flow rate in the chamber for ultrasonic concentration and selection, we compared the radiation pressure force at the various ultrasound energy parameters for cells and the Stokes force appearing with the cultivation's medium 
flow. The results of theoretical calculations are presented as the plots with the average energy density of the ultrasonic field and the linear velocity of the flow of the cell suspension as coordinate axes and as the Table 1. Straight lines in the plots separate the regions of cell concentration, separation, and washout. The slope of these straight lines is a characteristic of a certain cell type. A comparison of such dependences for two cell types' shows that there exists a region in which cells of one type will be washed away, and cells of another type will be concentrated (Figure 2).

Thus, we can determine the parameters of the ultrasonic field and velocities of the flow of the cell suspension, at which both cell concentration and separation will be observed. To test the chosen boundary conditions, we made a number of experiments to study cell concentration in the chamber for ultrasonic separation for different rates of pumping. Thus, in each experiment, we were fixed number of the cells.

The result is (Figures 3-5), dependence the concentration of cells from the suspension at the flow rate and of the average energy density of $1.6 \times 10^{-5} \mathrm{~J} / \mathrm{cm}^{3}$ and $2.5 \times$ $10^{-5} \mathrm{~J} / \mathrm{cm}^{3}$. The horizontal line is the theoretical expectation of our experiment. That is a reflection of the number of cells, which we expect to see in a cell for ultrasonic separation after passing the cell suspension through the chamber. We see that for all cell types at certain speeds leveling suspension begins washing cells from the chamber. The dashed vertical line indicates the speeds that were identified in Figure 2 as the boundary.

By increasing the pumping rate by a large amount of cells starts washed out of the camera, as the condition fails $F_{r} \geq F_{S 1}$. In Figure 2 marked the point at which speeds, there will be a limit of concentration/washout for

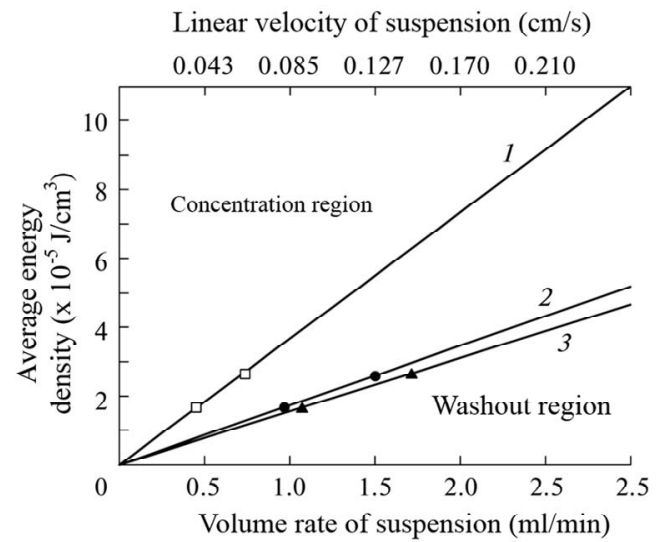

Figure 2. Determination of the concentration, separation, and washout regions for three cell types in the coordinate system of the average energy density of the standing ultrasonic wave and the linear velocity of the cell suspension flow. Theoretically calculated region separation lines $(1,2,3)$ and experimental data (symbols) for (1) yeast cells, (2) rat erythrocytes, and (3) guinea-pig erythrocytes.

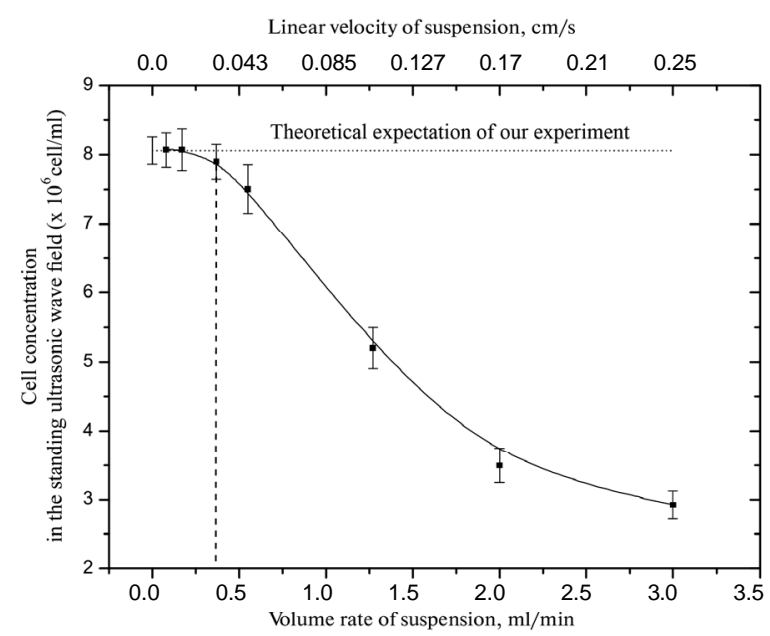

(a)

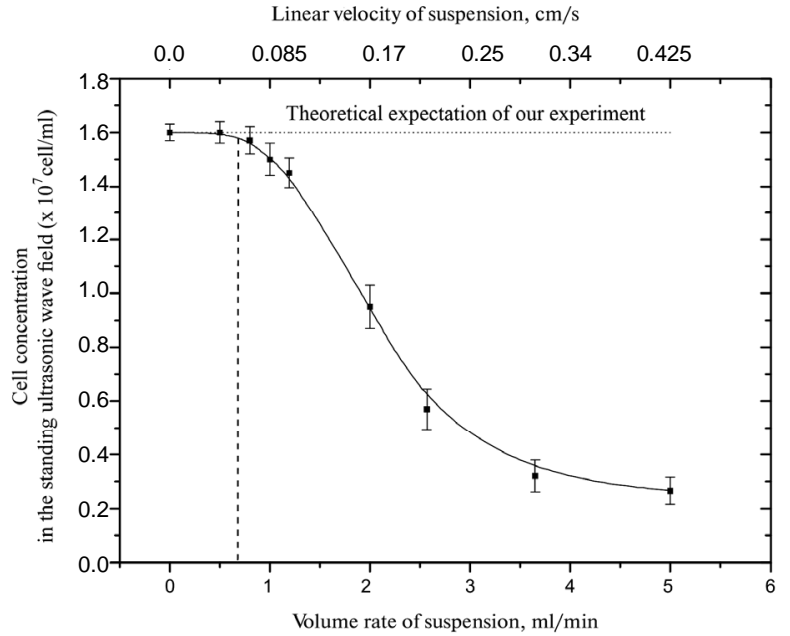

(b)

Figure 3. Dependence of the concentration of yeast cells in a suspension of the slurry from flow rate in a standing ultrasonic wave to the average energy density of (a) 1.6 and (b) $2.5\left(\times 10^{-5} \mathrm{~J} / \mathrm{cm}^{3}\right)$.

Table 1. Table type styles (Table caption is indispensable).

\begin{tabular}{ccccc}
\hline \multirow{2}{*}{$\begin{array}{c}\text { Object } \\
\text { of study }\end{array}$} & \multicolumn{4}{c}{ Average energy density $\left(\mathrm{J} / \mathrm{cm}^{3}\right)$} \\
\cline { 2 - 5 } & \multicolumn{3}{c}{$1.6 \times 10^{-5}$} & \multicolumn{2}{c}{$2.5 \times 10^{-5}$} \\
\cline { 2 - 5 } $\mathrm{Vr}^{\mathrm{a}}$ & 0.4 & 0.034 & 0.7 & 0.0595 \\
\hline Yeast cells & 0.9 & 0.0765 & 1.4 & 0.119 \\
$\begin{array}{c}\text { Wistar rat } \\
\text { erythrocytes }\end{array}$ & 1.1 & 0.0935 & 1.7 & 0.145 \\
$\begin{array}{c}\text { Guinea-pig } \\
\text { erythrocytes }\end{array}$ & & & & $\mathrm{Lv}$ \\
\hline
\end{tabular}

${ }^{\mathrm{a}}$ Volume rate of suspension $(\mathrm{ml} / \mathrm{min})$; ${ }^{\mathrm{b}}$ Linear velocity of suspension $(\mathrm{cm} / \mathrm{c})$.

the studied types of cells.

For yeast cells, with an average energy density of 1.6 $\times 10^{-5} \mathrm{~J} / \mathrm{cm}^{3}$ washout occurs at a flow rate a suspension at more than of $0.4 \mathrm{ml} / \mathrm{min}$, and at $2.5 \times 10^{-5} \mathrm{~J} / \mathrm{cm}^{3}$ more than $0.7 \mathrm{ml} / \mathrm{min}$. 


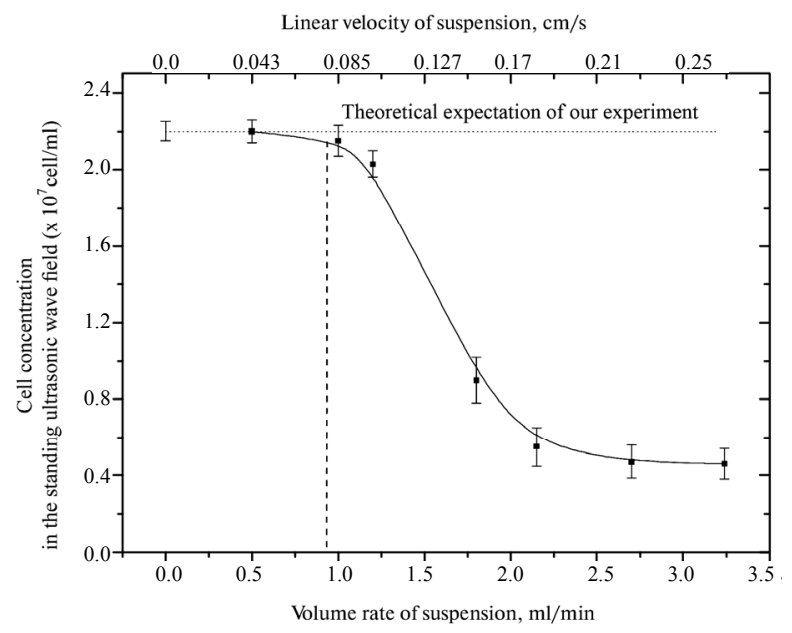

(a)

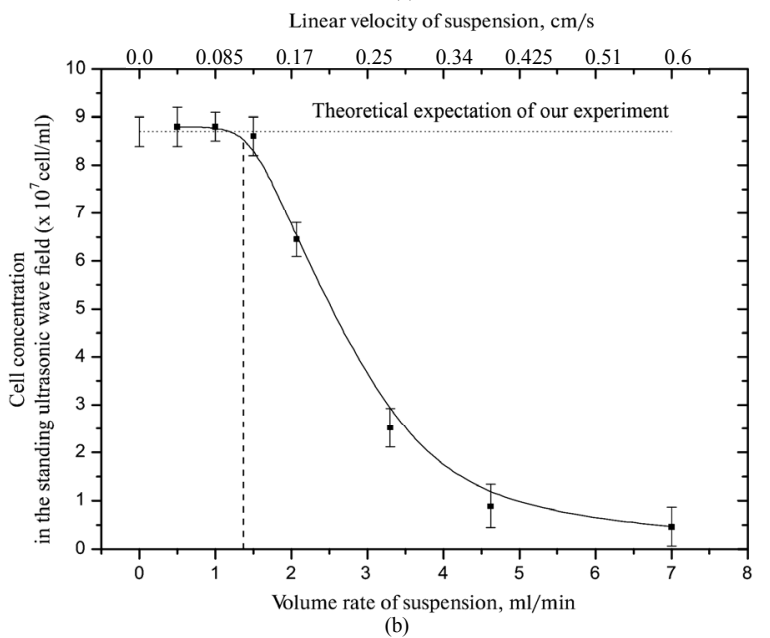

Figure 4. Dependence of the erythrocyte suspension from the flow velocity of the suspension in a standing ultrasonic wave to the average energy density of (a) 1.6 and (b) 2.5 (* $10^{-5} \mathrm{~J} / \mathrm{cm}^{3}$ ).

For red blood cells of rats with high energy density 1.6 $\times 10^{-5} \mathrm{~J} / \mathrm{cm}^{3}$ washout occurs at a flow rate a suspension at more than $0.9 \mathrm{ml} / \mathrm{min}$, and at $2.5 \times 10^{-5} \mathrm{~J} / \mathrm{cm}^{3}$ - more than $1.4 \mathrm{ml} / \mathrm{min}$. For red blood cells of guinea pigs with an average energy density of $1.6 \times 10^{-5} \mathrm{~J} / \mathrm{cm}^{3}$ washout observed at a flow rate a suspension at more than of 1.1 $\mathrm{ml} / \mathrm{min}$, and at $2.5 \times 10^{-5} \mathrm{~J} / \mathrm{cm}^{3}$ more than $1.6 \mathrm{ml} / \mathrm{min}$. The experimental results confirm our theoretical calculations made earlier and marked in Figure 2.

By the nature of the concentration of the studied cells from the flow rate of the medium, it is clear that to limit speed cell concentration does not change. And then drops sharply the number of cells in the chamber, and an increase in the flow rate to zero.

\section{Conclusion}

At the appearance of the method of ultrasound separation for isolation, separation and concentration of cells in sus-

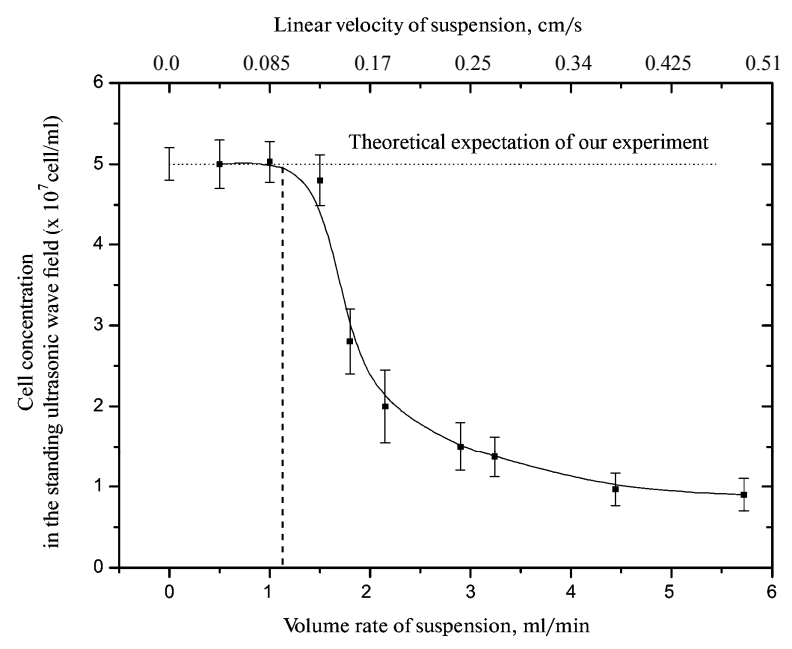

(a)

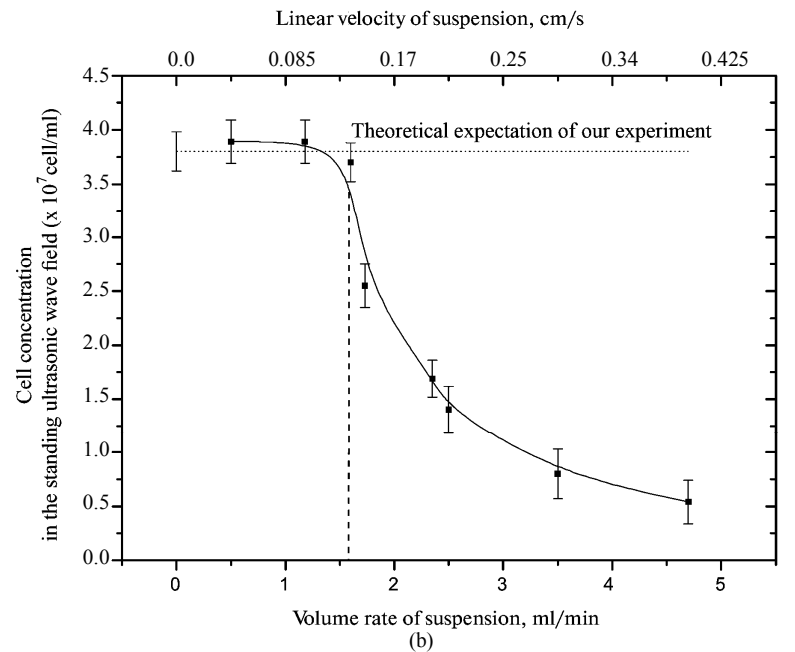

Figure 5. Dependence of the concentration of red blood cells of guinea pigs in the suspension from the flow rate of the suspension in a standing ultrasonic wave to the average energy density of (a) 1.6 and (b) $2.5\left(\times 10^{-5} \mathrm{~J} / \mathrm{cm}^{3}\right)$.

pension has solved the problem of fast, effective and non-invasive ways to work with the cells in the dynamic mode. Our previous theoretical studies have revealed a number of relationships that are important for the application of the method of ultrasound separation. In addition, these studies have a more accurate and complete description of the mechanisms of action of forces on cells in suspension in a standing ultrasonic wave and provide boundary conditions for the concentration and separation of cells using the method of longitudinal ultrasonic separation. The increase in the flow velocity of the medium above the theoretically calculated values for cell in a standing ultrasonic wave causes them to washing away from the camera separation ultrasound. The results of this work-is the next step to a controlled ultrasonic separation and concentration of particles of different types and sizes. In addition, the experiments to described in this paper, 
fully confirmed the theoretical calculations made by the authors.

\section{REFERENCES}

[1] K. Sollner and C. Bondy, "The Mechanism of Coagulation by Ultrasonic Waves," Transactions of the Faraday Society, Vol. 32, 1936, pp. 616-623.

[2] F. Fittipaldi, "Particle Coagulation by Means of Ultrasonics," Acustica, Vol. 41, No. 4, 1979, pp. 263-266.

[3] M. Mantysalo and E. Mantysalo, "Extraction and Filtering in Ultrasonic Field: Finite Element Modeling and Simulation of the Processes," Ultrasonics, Vol. 38, No. 1, 2000, pp. 723-726. doi:10.1016/S0041-624X(99)00163-8

[4] K. Yasuda, S. Umemura and K. Takeda, "Concentration and Fractionation of Small Particles in Liquid by Ultrasound," Japanese Journal of Applied Physics, Vol. 34, No. 1, 1995, pp. 2715-2720. doi:10.1143/JJAP.34.2715

[5] Z. I. Mandralis and D. L. Feke, "Continuous Suspension Fractionation Using Acoustic and Divided Flow Fields," Chemical Engineering Science, Vol. 48, No. 23, 1993, pp. 3897-3905. doi:10.1016/0009-2509(93)80368-Z

[6] Z. I. Mandralis and D. L. Feke, "Fractionation of Suspensions Using Synchronized Ultrasonic and Flow Fields," AIChE Journal, Vol. 39, No. 2, 1993, pp. 197-206. doi:10.1002/aic.690390202

[7] Z. I. Mandralis, W. Bolek, W. Burger, E. Benes and D. L. Feke, "Enhanced Synchronized Ultrasonic and Flow-Field Fractionation of Suspensions," Ultrasonics, Vol. 32, No. 2, 1994, pp. 113-121. doi:10.1016/0041-624X(94)90019-1

[8] S. Gupta, D. L. Feke and I. Manas-Zloczower, "Fractionation of Mixed Particulate Solids According to Compressibility Using Ultrasonic Standing Wave Fields," Chemical Engineering Science, Vol. 50, No. 20, 1995, pp. 32753284. doi:10.1016/0009-2509(95)00154-W

[9] S. Peterson, G. Perkins and C. Baker, "Development of an Ultrasonic Blood Cell Separator," IEEE Proceedings of the Eighth Annual Conference of Engineering in Medicine \& Biology Society, 1986, pp. 154-156.
[10] G. Whitworth, M. A. Grundy and W. T. Coakley, "Transport and Harvesting of Suspended Particles Using Modulated Ultrasound," Ultrasonics, Vol. 29, No. 6, 1991, pp. 439-444. doi:10.1016/0041-624X(91)90073-H

[11] F. Trampler, S. A. Sonderhoff, P. W. S. Put, D. G. Kilburn and J. M. Piret, "Acoustic Cell Filter for High Density Perfusion Culture of Hybridoma Cells," Biotechnology, Vol. 12. No. 3. 1994, pp. 281-284. doi:10.1038/nbt0394-281

[12] O. Doblhoff-Dier, T. Gaida, H. Katinger, W. Burger, M. Groschl and E. Benes, "A Novel Ultrasonic Resonance Field Device for the Retention of Animal Cells," Biotechnology Progress, Vol. 10. No. 4. 1994, pp. 428-432.

[13] T. N. Pashovkin and D. G. Sadikova, "Cell Exfoliation, Separation, and Concentration in the Field of a Standing Ultrasonic Wave," Acoustical Physics, Vol. 55, No. 4-5, 2009, pp. 584-593. doi:10.1134/S1063771009040150

[14] D. G. Sadikova, A. A. Andreev, A. N. Shkidchenko and T. N. Pashovkin, "Dynamics of Cells Concentration in a Standing Ultrasonic Wave," Biomedical Tekhnology and Electronics, No. 8-9, 2006, pp. 95-99.

[15] T. N. Pashovkin, D. G. Sadikova, M. S. Pashovkina and G. V. Shil'nikov, "The Use of Ultrasonic Standing Wave in Biological Research and Cell Technologies," Bulletin of Experimental Biology and Medicine, No. 3, 2007, pp. 133-138.

[16] A. A. Andreev, D. G. Sadikova and T. N. Pashovkin, "The Effect Forces on the Yeast Cells (Saccharomyces cerrevisiae) in a Standing Ultrasonic Wave," Bulletin of New Medical Tekhnology, Vol. 14, No. 2, 2007, pp. 1215.

[17] V. A. Shutilov, "Foundations of Ultrasound Physics," Leningrad University Leningrad, 1980, p. 104 (in Russian).

[18] N. N. Knyaz'kov and G. V. Shil'nikov, "Ultrasonic Concentration Cell and Tissue Cultures," Bulletin of Experimental Biology and Medicine,, No. 3, 1996, pp. 312-316.

[19] N. N. Knyaz'kov, E. D. Makarova and A. D. Rabizhanovich, "Ultrasonic Flow-Through Fractionation of Different Nature Particles," Scientific Instrument, Vol. 19, No. 1, 2008, pp. 40-55. 